\title{
A propos de Jean-Louis Prévost (1790-1850) \\ (Documents inédits)
}

Par Jean Théodoridès

«J'ai vu l'admirable

Prévost à Genève»

(Stendhal)

A l'occasion de la présente commémoration, il fallait, semble-t-il, que soit prononcé le nom de Jean-Louis Prévost (1790-1850) dont l'œuvre biologique et médicale honore la ville de Genève dont il était originaire.

J'ajouterai que Prévost m'intéresse triplement: en tant que biologiste, historien de la Biologie et stendhalien, car il fut le médecin et l'ami d'Henri Beyle, aspect de lui que j'ai déjà étudié ailleurs (Théodoridès 1969). Comme biologiste et physiologiste, Prévost est surtout connu par ses recherches avec Jean-Baptiste Dumas (1800-1884) sur la génération animale (rôle des spermatozoïdes dans la fécondation de l'œuf qu'ils furent les premiers à voir chez les Mammifères, avant von Baer (de Morsier 1965), l'urémie expérimentale (Mani 1963), la contraction musculaire, la transfusion sanguine, etc. (Buess 1974).

Il a de plus travaillé sur la régénération du tissu nerveux, sur la nutrition du fœtus (avec A. le Royer), sur la digestion gastrique (avec A. Morin) et fut un grand praticien bien que n'ayant presque rien publié en médecine clinique. Ackerknecht (1966) le qualifie «aussi grand comme praticien que comme expérimentateur microscopiste et biochimiste » et le met avec raison sur le même plan que Johannes Müller et Purkinje.

L'œuvre de J.L. Prévost étant bien connue et mise en valeur, je voudrais aujourd'hui donner quelques indications sur divers manuscrits de lui que j'ai eu l'occasion de trouver dans plusieurs fonds d'archives tant en Suisse qu'en France et en Grande-Bretagne. Deux des documents recensés ici seront publiés en annexe de la présente communication.

I. Genève

A) Bibliothèque Publique et Universitaire

Ms. 2663 (fols. 237-254) : 9 lettres de J. L. Prévost à L.A. Gosse ${ }^{1}$ (1815-1825).

Ces lettres écrites d'Edimbourg, Paris et Genève donnent des renseignements intéressants sur les études et les projets futurs de recherche du jeune savant. J'en ai publié un court extrait (Théodoridès 1969). 
Ms. 2146: Correspondance de P.F. Tingry ${ }^{2}$ : lettre de J. L. Prévost du 23 août 1820 annonçant ses examens devant la Faculté (de Genève ?).

Suppl. 1056 (Ms. 1925/33): Lettre de J.L. Prévost à Oersted ${ }^{3}$ du 13 juin 1826 lui annonçant l'envoi de son mémoire sur la génération des moules.

Ms. Fr. 306, pièce 28 : Lettre de J. L. Prévost à son oncle Guillaume Moultou du 24 février 1826, à propos de la fondation d'un asile d'aliénés à Genève.

Ms. fr. 3786 : Lettre de J.L. Prévost à Jacob Duval ${ }^{4}$ du 29 janvier 1841 lui annonçant le décès de son père Jean-René Prévost (né le 4 septembre 1759) survenu le jour même.

\section{B) Musée d'Histoire des Sciences}

(Je dois les indications suivantes à l'obligeance de M. Marc Cramer.)

Z 70/9 De l'emploi du muriate de platine dans les maladies nerveuses. Lu à la Soc. cantonale le 13 avril 1831.

Z 70/1 2 lettres à André Matthey. s.l. 1 juil. 1829 et 8 août 1831. Envoi des malades à St-Gervaix.

Z 70/8 Lettres à X (sans doute le Dr. Peschier) ${ }^{5}$ s.l. $1^{\text {er }}$ mars 1832 . Donne sa démission de la Société médicale.

Z 105 Observation de tétanos idiopathique (21 fols.). Note sur une tumeur cancéreuse (12 fols., 4 figs.). Voyage médical sur les bords du Rhin (25 fols.).

Ce dernier manuscrit non daté doit être par son contexte postérieur à 1843. Au cours de son voyage, Prévost visita successivement Bâle (dont il décrit l'Hôpital général), Fribourg-en-Breisgau, Baden-Baden, Karlsruhe, Heidelberg (où il rencontra L.Gmelin et J. Henle); à propos de cette dernière visite, Prévost dit avoir vu une importante collection d'injections microscopiques et de préparations anatomiques et s'exclame à ce sujet : «Quand je parle de médecine physiologique, je n'entends pas celle de Broussais!»

Dessins divers: Vues de la Jamaïque (novembre 1826) probablement données à Prévost par un ami. Viscères de la daurade et de la bécune. Dessins de pustules vaccinales (en couleurs). Embryogénie du cabiai (Capibara capibara), de la truite (1847). Spermatozoïdes et ovules (chienne). Fibres musculaires de divers animaux : limace, Carabus, escargot, salamandre grenouille, lézard, couleuvre, séchot, truite, Oiseaux (Fringilla, Corvus, Hirundo), Mammifères (cabiai, bœuf, mouton, chat) etc.

Z 172 Physiological Objects : 8 albums de notes illustrées de nombreux dessins (1843-1847). 1 album $13 \times 20 \mathrm{~cm} ; 7$ albums $24 \times 18 \mathrm{~cm}$. 
Ces albums bourrés de notes (écrites en anglais, langue que Prévost connaissait parfaitement, ayant fait ses études médicales à Edimbourg) accompagnant de très nombreux dessins en noir et en couleurs témoignent de la très grande activité de J.L.Prévost comme anatomiste et microscopiste.

On y trouve, étudiées sous divers points de vue, des espèces appartenant aux groupes les plus divers du règne animal : fibres musculaires d'Insectes (carabe, papillon), de Crustacés (écrevisse), branchies de Poissons (lotte), de têtards d'Amphibiens (grenouille, triton, etc.) dont il figure également le développement du tube digestif et de l'appareil circulatoire; embryologie du poulet avec d'innombrables dessins; étude de l'appareil génital de la brebis et développement du fœtus d'agneau, du lapin, du cabiai, etc.

Ce qui frappe l'observateur dans ces albums, c'est la fréquence avec laquelle leur auteur figure tel ou tel détail anatomique ou histologique. C'est ainsi que l'on trouve représentées plus d'une dizaine de fois l'anatomie viscérale du têtard de grenouille ou la fibre musculaire du carabe, comme si Prévost éprouvait un véritable plaisir à reproduire à plusieurs reprises de tels détails dont la structure avait dû le frapper ou l'intéresser tout particulièrement.

Z 1/21 A.P.de Candolle

Z 1/34 J. B. Dumas

Z 70/1 André Matthey

Il s'agit de lettres adressées à J.L. Prévost; celle de J.B. Dumas écrite au printemps de 1840 concerne les recherches d'Henri Milne-Edwards sur la spermathèque des seiches; un fragment en a été publié par le Dr. J. Olivier (Journal de Genève, 21 septembre 1950) avec une seconde datée du 31 août 1840 provenant des archives Vieusseux.

Notons pour terminer que la lettre à Alphonse Favre signée J. L. Prévost datée de Londres, 16 mars 1846 et conservée sous la cote Z 176/1 No 45 n'est pas du biologiste, mais de son homonyme (1796-1852) agent et consul de Suisse à Londres, fils de Pierre Prévost (1751-1839).

\section{Paris (Archives de l'Académie des Sciences)}

Dans les papiers de J. B. Dumas se trouvent quatre lettres adressées par J. L. Prévost à son collaborateur et ami, datées respectivement des 18 mai 1839, 6 janvier 1840, 12 mars 1841, 11 décembre 1842.

Du point de vue scientifique ce sont la première et la dernière lettre qui sont les plus intéressantes. Dans la première, Prévost prie Dumas de rectifier leurs 
observations antérieures sur la morphologie des spermatozoïdes d'Amphibiens (grenouille, crapaud, salamandre) et celui-ci fit publier l'essentiel de ces rectifications $^{6}$. Prévost ajoute en terminant dans la seconde lettre : «Je suis sûr du fait, regardez-le de votre côté, il ne faudrait pas laisser à Du Trochet le plaisir de le dire avant nous. J'ai lu son livre ${ }^{7}$ où il ne cite de nous que les choses sur lesquelles il peut nous prendre en faute.»

Dans la troisième lettre, Prévost annonce à son ami la mort de son père et fait preuve d'un bel amour filial lorsqu'il dit :

«Mon père est mort à la suite d'une longue et pénible maladie et cette séparation pour être prévue n'en a pas été moins douloureuse ; non seulement il avait été toujours pour moi un excellent père, mais depuis que j'avais été en âge de raison, il m'avait traité comme un ami. Heureusement, il m'a été donné de pouvoir le soigner pendant sa vieillesse et ce souvenir, je le préfère à toute chose.»

Ceci confirme la dévotion bien connue de J.L. Prévost pour son père Jean-René Prévost (1759-1841).

La quatrième lettre concerne des expériences sur l'électricité animale et, vu son grand intérêt scientifique, elle figure en annexe du présent exposée ${ }^{8}$.

\section{Londres}

\section{A) Archives de la Royal Society}

1822-1825, vol. 11, A.P. 11/9. Manuscrit intitulé «De la génération chez les Poissons» daté de Genève 25 avril 1824.

Il s'agit d'un ensemble de 20 folios petit format accompagnés d'une planche en couleurs (Heyland pinxit) consacrés au développement de Cottus gobio (chabot commun), poisson d'eau douce. Prévost y décrit tout d'abord l'appareil génital mâle (testicules, spermatozoïdes) et femelle (ovaires, œufs) puis le développement de l'œuf. Il note l'aspect du fœtus de 6 et 8 millimètres et décrit les ébauches cérébrales et cardiaques.

Ce mémoire inédit s'inscrit dans les recherches entreprises de 1821 à 1827 par Prévost et Dumas sur la reproduction et la génération de divers Vertébrés (Poissons, Amphibiens, Mammifères) et Invertébrés (Mollusques Lamellibranches et Gastéropodes) auxquelles nous avons fait allusion plus haut. Il a du être envoyé par son auteur à la Royal Society qui ne l'a jamais publié.

\section{B) Wellcome Historical Medical Museum and Library}

(Autographes No 67802) ${ }^{9}$ : Lettre à Mademoiselle Peschier (Plein Palais, Genève) du 15 mai 1846. 
Ce document est intéressant en ce qu'il nous montre J.L. Prévost clinicien, écrivant ici à la sœur d'un malade atteint probablement de tuberculose, si fréquente à cette époque.

Nous le donnons en Appendice II de la présente communication. Il y a certainement d'autres papiers inédits (manuscrits, lettres etc.) de J. L. Prévost dans divers fonds d'archives européens et cette communication ne constitue qu'une introduction préliminaire à une étude plus importante que nous entreprendrons peutêtre dans un avenir prochain.

\section{Appendice I}

Lettre de J.L. Prévost à J.B.Dumas (11 décembre 1842) 10

(Archives de l'Académie des Sciences, Papiers J. B. Dumas)

\section{Mon cher Dumas}

Votre lettre m'a fait grand plaisir et j'ai de suite répété les expériences que vous m'indiquiez avec le pigeon et la grenouille. $-1^{\circ}$ Quand on a divisé le pectoral on a un courant qui va de la partie supérieure du muscle à l'inférieure. $-2^{\circ} \mathrm{Si}$ l'on applique deux plaques de platine soudées aux deux extrémités des fils du galvanomètre aux deux portions divisées du muscle, l'on a un courant de dix à douze degrés, dans le sens du haut du muscle, à sa partie inférieure. $-3^{\circ} \mathrm{Si}$ l'on introduit les petites lames dans le muscle sans le diviser l'on a un faible courant qui va de la partie inférieure du muscle, si l'on divise alors le muscle entre les deux lames, le courant change de direction et j'ai même obtenu alors jusqu'à douze degrés au galvanomètre (comme dans l'autre cas). $-4^{\circ}$ Sans employer une excitation galvanique vous pouvez faire contracter la grenouille dont on a placé les nerfs sur le bord de la plaie faite dans le muscle d'une autre, pour cela les nerfs coupés à leur sortie de la mœlle épinière et placés sur les bords de la plaie comme nous l'avons dit, l'on fait contracter la $1^{\text {re }}$ grenouille en passant un stilet [sic] dans la mœlle épinière l'on a une violente contraction et la seconde grenouille se contracte. Je vais maintenant étudier sur la mœlle épinière d'un animal fait de la même manière que nous l'avons fait pour les muscles et je vous communiquerai mes résultats. Toutes ces expériences sont curieuses, mais n'expliquent pas encore la contraction et il faut en revenir à nos idées : pour essayer d'éclaircir ce sujet, j'ai fait faire l'instrument suivant et je vais l'essayer si l'expérience réussit je vous la communiquerais; j'ai fait faire vingt quatre hélices dont les fils sont enroulés en sens inverse sur chacune d'elles. Je placerai les jambes de mes grenouilles chaque jambe dans l'hélice correspondante et ainsi de suite. Je préparerai l'ex- 
périence de manière à faire contracter toutes les grenouilles simultanément, ce qui est très facile puis plaçant une grenouille préparée sur les deux bouts terminaux des fils des hélices je verrai s'il se forme un courant d'induction dans les spirales, ce qui indiquerait des courants circulaires dans le muscle contracté. Si vous pouviez répéter l'expérience avec les moyens dont vous disposez, j'en serais bien aise.

J'ai fait sur les insectes une autre expérience qui serait contre les courants circulaires, quand on fait contracter le muscle d'un insecte, on voit que les fibrilles qui composent le faisceau musculaire sont disposées ainsi et tous ces points se rapprochent dans plusieurs cas avec une rapidité incroyable et isolément. Par l'expérience de Matteucci ${ }^{11}$ on déduirait que le courant propre des muscles chargerait le cerveau et la mœlle épinière en condensateur dont partirait le courant à volonté.

Je finis ma lettre avec mon papier, elle est bien embrouillée, mais c'est que votre ami est loin d'être bien portant.

$$
\text { V [otre] a[mi] J. L. Prévost }
$$

$11-12$

[18] 42

Appendice II

Lettre de J.L.Prévost à Mademoiselle Peschier ${ }^{12}$ (15 mai 1846)

Mademoiselle

Mademoiselle Peschier

Plain Palais

Mademoiselle,

J'ai attentivement lu la lettre de Monsieur le Dr Ratier et celle de Monsieur votre frère. Je n'ai pas vu des effets bien satisfaisants de l'huile de foie de morue. C'est pourtant un remède qui jouit d'une certaine considération; s'il augmente l'appétit, il n'arrête pas les digestions alors il convient et l'on peut en prescrire l'usage - les vapeurs du goudron réussissent plutôt dans le catarrhe chronique que dans les affections proprement dites du poumon du genre tuberculeux. Elles augmentent la toux, je préfèrerais des fumigations d'eau avec de très légères doses d'ammoniaque. Quant aux Eaux Bonnes ${ }^{13}$ comme nous le disions, elles ne conviennent pas là où il y a trop d'irritation pulmonaire, elles activent l'inflammation dans tous les cas. C'est un remède qui comme les eaux du Mont Dore ${ }^{14}$ doit être dirigé par le médecin de très près. 
Bien que je n'aye pas l'honneur de connaître Monsieur votre frère, veuillez lui exprimer combien je suis peiné de sa position.

Veuillez, Mademoiselle, agréer mes respects empressés,

$15-5$

J. L. Prévost

[18] 46

\section{Notes}

${ }^{1}$ Il s'agit de Louis-André Gosse (1791-1873) médecin genevois qui fondera avec J.L.Prévost et Dupin le dispensaire des médecins et le Journal de Genève (1829).

2 Pierre-François Tingry (1743-1821) professeur de chimie à Genève.

${ }^{3}$ Le célèbre chimiste et physicien danois H.C. Oersted (1777-1851).

${ }^{4}$ Jacob-Louis Duval (né en 1797) juriste puis député à Genève.

${ }^{5}$ Voir plus loin en ce qui concerne cette famille. D'après la date il ne peut s'agir de Jean Peschier décédé en 1831.

${ }^{6}$ J.L.Prévost, Recherches sur les animalcules spermatiques, C.R. Acad. Sci. 11 (1840), p. 907-908.

7 Il s'agit certainement des "Nouvelles recherches sur l'œuf des animaux vertébrés», Mém. Soc. Méd. Emulat. 9 (1826), p. 11-64, d'Henri Dutrochet (1776-1847). Le catalogue de la librairie de l'Abbaye, 27 rue Bonaparte, Paris $6^{\mathrm{e}}$, Bulletin $\mathrm{N}^{\mathrm{o}} 47$, donne sous le $\mathrm{N}^{\circ} 80$ une lettre de J.L.Prévost à Dutrochet datée de Genève 9.5.1827 où il le remercie pour son ouvrage.

Dans leur livre récent : Henri Dutrochet (Henri du Trochet 1776-1847), Paris (Blanchard) 1975, J. et T. Schiller mentionnent (p. 17) que Dutrochet se rendit en 1826 à Genève pour y rencontrer J.L. Prévost.

${ }^{8}$ Monsieur G. Dumas Milne-Edwards, descendant de J. B. Dumas, m'a aimablement signalé qu'il y a dans la propriété familiale de L'Aulnaie (près de Nogent-le-Rotrou, Eure-etLoir) une série de dessins représentant l'évolution de l'embryon de poulet et de lapin étudiée par Dumas et Prévost et qui seraient peut-être de la main de ce dernier.

${ }^{9}$ Nous remercions très vivement son ex-directeur, le Dr F.N.Poynter, qui nous a procuré une copie de cette lettre et nous a autorisé à la publier. En haut du feuillet portant le nom de la destinataire, on peut lire d'une autre écriture: «Dr Prévost, de Genève, collaborateur de Mr Dumas dans les travaux sur le sang de ce célèbre chimiste».

${ }^{10} \mathrm{Je}$ remercie MM. les Secrétaires Perpétuels de l'Académie des Sciences qui m'ont autorisé à publier cette lettre.

${ }^{11}$ Le physiologiste italien Carlo Matteucci (1811-1868).

${ }^{12}$ La famille Peschier très connue à Genève a donné plusieurs personnes célèbres; théologien, astronome et mathématicien, pharmacien, médecin: Jean Peschier (1774-1831). Je remercie Mr. Georges de Morsier qui m’a communiqué ce renseignement.

${ }^{13}$ Il s'agit de la station thermale des Basses-Pyrénées (arrondissement d'Oloron).

${ }^{14}$ Station thermale (eaux arsenicales) du Puy-de-Dôme. 
Ackerknecht, E. H., La médecine à Genève surtout dans la première moitié du XIX ${ }^{\mathrm{e}}$ siècle. In : Verh. XIX. Int. Kongr. Gesch. d. Med. (Basel 1964), Basel (Karger) 1966, p. 420-425.

Brunner, V., Der Genfer Arzt Jean-Louis Prévost (1790-1850) und sein Beitrag zur Entwicklungsgeschichte und Physiologie. Thèse Méd. Zürich 1966, 35 p.

Buess, H., Un pionnier de la biologie moderne : Jean-Louis Prévost (1790-1850). Méd. et Hyg., 1974, $\mathrm{N}^{\circ} 1118$, p. 1578-1580.

Mani, N., La découverte de l'urémie expérimentale par Jean-Louis Prévost et Jean-Baptiste Dumas, Genève 1821. Méd. et Hyg., 1963, N 593 , p. 408-409.

Morsier, G. de, Contribution à l'histoire de la génétique. L'œuvre des biologistes genevois Colladon, Vaucher, Prévost et Fol. Physis 7 (1965), p. 489-516.

- Jean-Louis Prévost (1790-1850) et la découverte de l'ovule des mammifères. Gesnerus 23 (1966), p. 117-121.

Olivier, J., «L'admirable Prévost» de Stendhal. Progr. Méd., 1954, p. 181-182.

Théodoridès, J., Jean-Louis Prévost médecin et ami de Stendhal. Stendhal Club, 1969, $\mathrm{N}^{\circ} 42$, p. 177-189.

\section{Summary}

After having briefly recalled the importance of J.L.Prévost's (1790-1850) biological and medical work, the author gives indications on some of his unpublished manuscripts (articles, notes and drawings, letters etc.) kept in various institutions and archives in Geneva, Paris and London. Two of them are published here.

Prof. Dr. Jean Théodoridès

16, Square Port-Royal

F-75013 Paris 